\title{
Resonating to the music of ubiquitination
}

\section{Sudha K Shenoy \& Robert J Lefkowitz \\ Beyond protein destruction: a new real-time assay to measure dynamic changes in ubiquitination promises to highlight the many other roles of this critical protein modification.}

Ubiquitination of cellular proteins generally marks them for destruction by the $26 \mathrm{~S}$ proteasome, a discovery that was recognized by the award of the 2004 Nobel Prize in Chemistry to Aaron Ciechanover, Avram Hershko and Irwin Rose ${ }^{1}$. More recent discoveries demonstrated that this protein modification, which consists of a posttranslational attachment of a 76-aminoacid ubiquitin peptide to the epsilon amino group of lysine, has a key role in regulating a growing list of cellular endocytic,
Figure 1 | Many consequences of protein ubiquitination. Lysine(s) or the Nterminal amino group of substrate proteins are covalently modified with ubiquitin (Ub) through the sequential activities of three enzymes: ubiquitin activating enzyme (E1), ubiquitin conjugating enzyme (E2) and ubiquitin ligase (E3). The ubiquitin tags on substrates can serve as (i) degradation signals for the destruction of substrate by the protease activity of the 265 proteasome (ii) maturation signals when the substrate is partially degraded or processed by the $26 \mathrm{~S}$ proteasome (iii) signals for intracellular trafficking or sorting into different populations of endosomes and (iv) modulators of signal transduction. The bottom of the figure shows the application of BRET technology to monitoring protein ubiquitination.

Sudha K. Shenoy is in the Department of Medicine and Robert J. Lefkowitz is in the Howard Hughes Medical Institute and in the Departments of Medicine and Biochemistry at Duke University Medical Center, Durham, North Carolina 27710, USA. e-mail: lefko001@receptor-biol.duke.edu trafficking and signaling functions. These discoveries have focused attention on the importance of dynamic alterations in the extent of ubiquitination of cellular regulatory molecules (Fig. 1). Thus, the report by Perroy et al. in this issue of Nature Methods, describing a bioluminescence resonance energy transfer (BRET)-based assay for in situ monitoring of moment-to-moment changes in protein ubiquitination, is timely indeed ${ }^{2}$. The authors demonstrate the power of the approach by examining a recently appreciated regulatory mechanism, the seven transmembrane (7TM) receptor-controlled ubiquitination of the multifunctional endocytic and signaling adaptor protein $\beta$-arrestin ${ }^{3}$.

Over the years scientists have been fascinated by the way proteins function as molecular machines by forming specific complexes in living cells. The study of dynamic interactions between proteins, as revealed by cellular colocalization experiments, has been aided by the development of fluorescent tags combined with substantial progress in microscopy. However, positive visualization of direct protein-protein dynamic interactions was not possible until a process known as fluorescence resonance energy transfer (FRET) was applied to the problem. FRET involves a distance-dependent direct energy transfer from an excited donor fluorophore to an acceptor fluorophore and provides a powerful method for live-cell studies ${ }^{4}$. For FRET to occur, the donor and acceptor fluorophores must be within 10-100 $\AA$ of each other. This will occur reliably only if the two proteins are closely associated. Furthermore, the donor fluorescence emission spectrum must overlap with the acceptor's absorption spectrum and the dipoles of the two molecules should be approximately parallel. Perroy et al. utilize a recent variation on FRET called BRET, where the donor molecule is the bioluminescent enzyme called luciferase. To measure the interaction between two proteins, luciferase, the donor, is fused to 'protein 1' and a fluorescent protein, the acceptor, is fused to 'protein 2'. When the luciferase enzyme is provided with a substrate such as DeepBlueC, blue light energy 
is released, and if the acceptor fluorophore is close enough there is a concomitant nonradiative transfer of energy from the donor to the acceptor. BRET occurs as the acceptor molecule captures this energy and fluoresces at a wavelength longer than that of the luciferase bioluminescence.

Like a FRET signal, the BRET signal serves as a measure of proximity or complex formation between the two proteins, but unlike FRET, which requires excitation of the donor fluorophore using an external light source, BRET relies on the selfcatalyzed release of light in the presence of a substrate molecule. Interaction and dimerization of circadian clock proteins and homo- and heterodimerization of 7TM receptors are examples of areas where BRET has provided valuable insights ${ }^{5-7}$. In an attempt to monitor the dynamic process of ubiquitination in living cells using BRET, Perroy et al. fused GFP to ubiquitin and fused luciferase to $\beta$-arrestin, whose ubiquitination was recently found to be dynamically regulated in response to stimulation of specific 7TM receptors ${ }^{3}$. They found that BRET resulting from energy transfer between the donor luciferase and the acceptor GFP moieties could specifically measure the dynamics of ubiquitination of $\beta$-arrestin.

Before these methods were developed, the measurement of protein ubiquitination routinely relied on isolating proteins of interest by cellular lysis and immunoprecipitation, followed by immunodetection with antibodies that specifically recognize ubiquitin. However, such methods are not very informative when the ubiquitinated species are short-lived, either because they undergo rapid proteasomal degradation, or because the modification is reversed by specialized deubiquitinating proteases. These problems often necessitate additional steps to inactivate such proteases to detect ubiquitinated proteins. In contrast, the newly developed BRET ubiquitination assay seems ideally suited for the detection of short-lived ubiquitinated species without perturbation of the cellular machinery.

A limitation of current BRET assays, however, is that they do not provide information about the cellular localization of the ubiquitinated proteins. On the other hand, a recently published paper by Fang and Kerppola addresses this issue via ubiquitin-mediated fluorescence complementation $(\mathrm{UbFC})^{8}$. These authors fused one-half of a fluorescent protein (YFP) to ubiquitin and the complementary half to Jun, a protein that regulates transcription and is considered a bona fide substrate for proteasomal degradation. When these two fusion proteins are coexpressed in cells, ubiquitination of Jun brings the two halves of YFP together to form a functional fluorescent molecule. It was found that Jun changes its localization from the nucleus to cytoplasmic lysosomes upon ubiquitination. However, the UbFC method is not well suited to monitor real-time ubiquitination processes, as it takes at least an hour for the fluorescence level of the conjoined YFP halves to become bright enough to be visualized. A FRET-based assay might better reveal the cellular location as well as dynamic modifications of proteins and therefore prove more useful for monitoring protein ubiquitination in the context of cellular localization.

Why are these new assays likely to be widely adopted? Since its original discovery in the context of nonlysosomal protein degradation, the process of protein ubiquitination has been implicated in an ever-growing list of cellular trafficking and signaling events (Fig. 1). Examples of traf- ficking functions involving ubiquitination include the vacuolar sorting of yeast receptors and the lysosomal targeting of mammalian cell surface receptors ${ }^{9}$. These functions depend on the covalent linkage of one or more ubiquitin moieties to the protein cargo, a mark that is recognized by specialized endocytic proteins, leading to their noncovalent binding to the ubiquitinated cargo. These noncovalent interactions are mediated by protein domains, which bind to hydrophobic surfaces on ubiquitin.

Even more recently, the realization that ubiquitination has previously unsuspected roles in signal transduction has opened new research avenues. This is a very young field, but already several examples have been described of ubiquitination regulating the activity of an upstream member of a signaling kinase cascade. For example, cellular signal transduction occurs via mitogen-activated protein kinases (MAPKs) which are enzymes that become activated in response to stress or cell surface receptor stimulation. MAPKs such as ERK (extracellular signal-regulated kinase) or JNK (Jun N-terminal kinase) phosphorylate and modulate the activities of several substrates leading to the regulation of such
Figure 2 | Roles of ubiquitination in 7TM receptor trafficking and signaling. Agonist stimulation of a 7TM receptor leads to phosphorylation of serine and threonine residues in the cytoplasmic domains. Activated and phosphorylated receptors recruit $\beta$-arrestin, which then brings the ubiquitination machinery into the receptor complex. The ubiquitin tags on mammalian receptors are crucial for the post-endosomal sorting of internalized receptors that leads to their degradation in lysosomes, whereas those on yeast receptors serve as internalization signals. In the mammalian system, receptor stimulation also leads to conformational changes in $\beta$ arrestins, after which $\beta$-arrestins are ubiquitinated. Agonist-dependent $\beta$-arrestin ubiquitination serves to promote receptor internalization. The kinetics of $\beta$-arrestin ubiquitination and deubiquitination differ with respect to different 7TM receptors. Upon $\beta_{2}$-adrenergic receptor stimulation, $\beta$-arrestins are transiently ubiquitinated, whereas stimulation of vasopressin V2 receptors leads to more sustained $\beta$-arrestin ubiquitination.

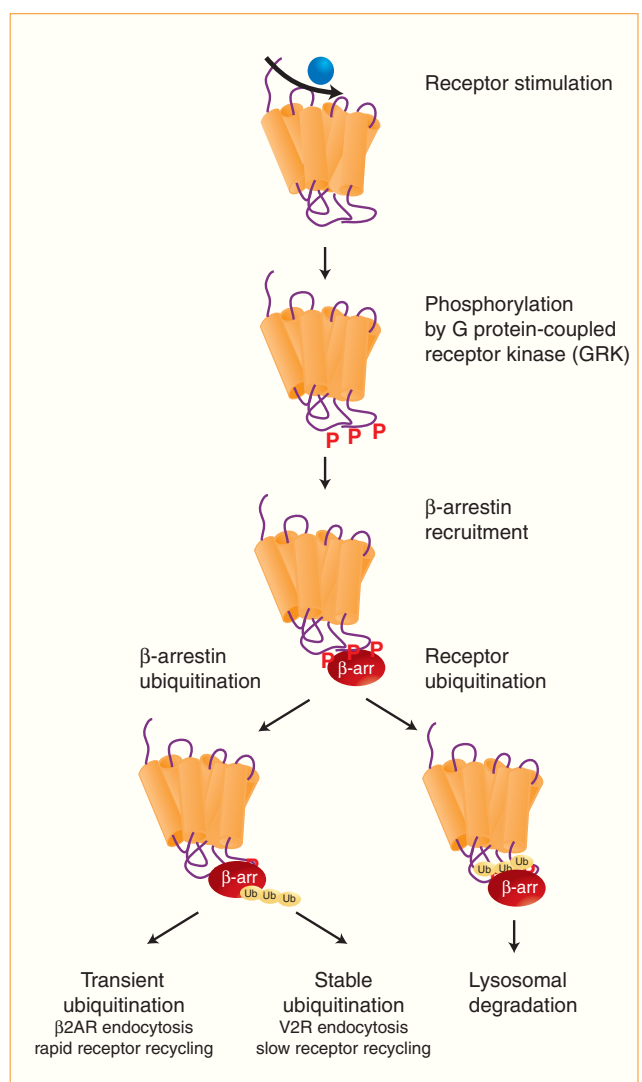


crucial cellular activities as mitogenesis, apoptosis and cell differentiation, among others. Ubiquitination has been shown to have inhibitory or stimulatory roles during signal transduction and cell response $\mathrm{e}^{10,11}$.

Ubiquitination may also have important regulatory roles at multiple steps of 7TM receptor signal transduction (Fig. 2) ${ }^{12}$. Agonist stimulation leads to ubiquitination of receptors, receptor associated proteins ( $\beta$-arrestin and $G$ protein-coupled receptor kinases) and downstream effectors (inositol $(1,4,5)$-trisphosphate receptors). Interestingly, receptor ubiquitination directs their sorting to lysosomes for downregulation, whereas $\beta$-arrestin ubiquitination is critical in promoting intracellular trafficking of the receptor ${ }^{13}$. $\beta$-arrestin ubiquitination is, for the most part, an agonist-driven modification, such that $\beta$-arrestin binding to activated receptors precedes ubiquitin attachment. Ubiquitination follows, presumably only after conformational changes occur in $\beta$ arrestin, which are induced by receptor interaction. Moreover, as confirmed by Perroy et al., $\beta$-arrestin quickly dissociates from the $\beta 2 A R$ as a result of its rapid deubiquitination, whereas with some other receptors, such as the $\mathrm{V} 2$ vasopressin receptor, $\beta$-arrestin remains stably associated and ubiquitinated ${ }^{2,3}$. Additionally, these differences in the kinetics of $\beta$-arrestin deubiquitination seem to determine the pattern of $\beta$-arrestin-induced receptor endocytosis. Receptors that provoke transient $\beta$-arrestin ubiquitination, such as $\beta 2 A R$, dissociate from it before internalization and recycle rapidly. Receptors that induce sustained ubiquitination, such as $\mathrm{V} 2$ vasopressin receptor $(\mathrm{V} 2 \mathrm{R})$, are internalized as receptor- $\beta$-arrestin complexes and remain in endosomes for prolonged periods before being recycled or degraded (Fig. 2) ${ }^{3}$.

Perroy et al. have also added a clever twist to their assay by simultaneously measuring two different BRET signals, one reflecting the ubiquitination of $\beta$-arrestin and the second reflecting formation of the receptor- $\beta$-arrestin complex. This provides the added advantage of correlating $\beta$-arrestin ubiquitination status with the extent of $\beta$-arrestin recruitment to the receptor in the same population of cells. By applying the same principles, one could evaluate the roles of $\beta$-arrestin ubiquitination in its interaction with many other signaling and endocytic partners. Such studies should rapidly lead to new insights into such fascinating questions as whether $\beta$-arrestin ubiquitination regulates its endocytic as well as signaling functions.

Thus, although it was discovered as a tag that marks proteins for destruction, research conducted in many laboratories around the world implicates ubiquitin as a modulator of many biological processes, such as DNA repair, kinase activation, gene transcription and trafficking of membrane proteins. In many cases, ubiquitinated proteins are not marked for death, but instead their activities are regulated or they are stabilized rather than degraded ${ }^{14}$. For several substrates, ubiquitination is closely coupled to other post-translational modifications such as phosphorylation and acetylation. Moreover, compared to small molecule-based modifications, ubiquitination provides greater versatility for possible structural and conformational variations, which facilitate substrate regulation. The 'ubiquitin era' has led to the discovery of several other ubiquitin-like molecules, such as small ubiquitin-like modifier (SUMO), related to ubiquitin (RUB), interferon stimulated gene 15 (ISG15) and autophagy 12-like protein (Apg12), whose functional roles are beginning to be appreciated ${ }^{15}$. It seems likely that BRET assays such as those described by Perroy et al. will greatly expedite the unraveling of the biological roles of these and other post-translational modifications related to ubiquitination.

1. Hershko, A., Ciechanover, A. \& Rose, I.A. Proc. Natl. Acad. Sci. USA 76, 3107-3110 (1979).

2. Perroy, J., Pontier, S., Charest, P., Aubry, M. \& Bouvier, M. Nat. Methods 1, 203-208 (2004).

3. Shenoy, S.K. \& Lefkowitz, R.J . J. Biol. Chem. 278, 14498-14506 (2003).

4. Miyawaki, A. \& Tsien, R.Y. Methods Enzymol. 327, 472-500 (2000).

5. Xu, Y., Piston, D.W. \& J ohnson, C.H. Proc. Natl. Acad. Sci. USA 96, 151-156 (1999).

6. Angers, S. et al. Proc. Natl. Acad. Sci. USA 97, 3684-3689 (2000).

7. Ramsay, D., Kellett, E., McVey, M., Rees, S. \& Milligan, G. Biochem. J. 365, 429-440 (2002).

8. Fang, D. \& Kerppola, T.K. Proc. Natl. Acad. Sci. USA 101, 14782-14787 (2004).

9. Schnell, J.D. \& Hicke, L. J. Biol. Chem. 278, 35857-35860 (2003).

10. Witowsky, J.A. \& J ohnson, G. L. J. Biol. Chem. 278, 1403-1406 (2003)

11. Wang, C. et al. Nature 412, 346-351 (2001).

12. Wojcikiewicz, R.J.. Trends Pharmacol. Sci. 25, 35- 41 (2004).

13. Shenoy, S.K., McDonald, P.H., Kohout, T.A. \& Lefkowitz, R.J . Science 294, 1307-1313 (2001).

14. Flick, K. et al. Nat. Cell Biol. 6, 634-641 (2004).

15. Yeh, E.T., Gong, L. \& Kamitani, T. Gene 248, 1-14 (2000). 